\title{
Heart Rate Variability in Response to Task Automation in Agricultural Semi-Autonomous Vehicles
}

\author{
Behzad Bashiri and Danny D. Mann*
}

Department of Biosystems Engineering, University of Manitoba, Winnipeg, MB R3T 5V6, Canada

\begin{abstract}
The goal of this study was to assess the sensitivity of the heart rate variability measure to variations in task difficulty and drivers' mental workload in agricultural semi-autonomous vehicles. 30 young participants with at least one year of tractor driving experience performed steering and implement control and monitoring tasks in a simulator study. Experiments were conducted using the tractor driving simulator (TDS) located in the Agricultural Ergonomics Laboratory at the University of Manitoba. Five levels of automation support from low to high were defined for the implement control and monitoring task. One-half of the participants performed a manual steering task while the other half drove the TDS in automatic steering mode, which only required them to monitor a mapping system and supervise the computer that was performing the steering task. A heart rate monitor was used to record the participants' heart rate. Time and frequency domain parameters of heart rate were analyzed. Some of the time and frequency domain parameters showed some sensitivity to workload variations, however, inconsistencies were observed in the results. The widely used HRV parameter, $0.1 \mathrm{~Hz}$ component of HRV, was not sensitive enough to differentiate mental workload levels when the drivers were involved in the task-loop.
\end{abstract}

Keywords: Agricultural vehicle, automation design, heart rate variability, mental workload.

\section{INTRODUCTION}

Operation of an agricultural vehicle on a field is a continuous task that requires much physical and mental effort. It usually involves primary tasks of i) driving and ii) implement control and monitoring. To enhance the performance of operation and reduce operators' workload, various automated systems have been introduced in these vehicles. Automatic steering systems, for instance, allow drivers to delegate the steering task while driving on a straight path toward a headland, allowing them to assign more attention to the implement control and monitoring task (ICMT). The ICMT also has been the subject of automation and its sub-tasks have been automated, partially or entirely. Although physical workload is reduced with these automated systems, mental workload remains unknown.

Mental workload is an important human factor in the driving context that has been the subject of many studies. It reflects perceptual and cognitive demands of tasks on limited mental resources [1]. However, it has been stated that other factors such as stress, fatigue and the level of motivation can also induce mental workload [2]. In the driving context, mental workload that is either too low or too high may lead to imperfect perception, insufficient attention and inadequate information processing [3]. Hence, the effects of automated systems in agricultural vehicles on drivers' mental workload should be identified.

Address correspondence to this author at the Department of Biosystems Engineering, University of Manitoba, Winnipeg, MB R3T 5V6, Canada; Tel: (204) 474-7149; Fax: (204) 474-7512;

E-mail: Danny.Mann@ad.umanitoba.ca
Driver's workload has been assessed through various methods [4]. As physiological measures of workload, heart rate (HR) and heart rate variability (HRV) have been shown to be sensitive to changes in physical and mental workloads [3]. According to Mulder et al. [5], mental efforts are associated with increased HR and decreased HRV. The term 'heart rate' describes the frequency of cardiac cycle in a person and usually is shown as the number of heart beats per minute $(\mathrm{bpm})$. Heart rate variability (HRV) refers to variations in heart rate, or more specifically, variations of intervals between consecutive $\mathrm{R}$ peaks of the heart beat waves. Heart beat is under the control of the autonomic nervous system that consists of two components: i) sympathetic nervous system (SNS) and ii) parasympathetic nervous system (PNS). The interaction between SNS and PNS results in variations in heart rate [6]. Under the conditions of acute time pressure and emotional strain, the PNS is suppressed and the SNS gets activated. Increased sympathetic activity results in increased heart rate. Conversely, PNS activation slows the heart rate.

To examine the fluctuations in autonomic nervous activity, power spectral analysis of heart rate variability (HRV) has been used instead of traditional cardiovascular measurements [7]. Mulder distinguished three different frequency bands for HRV including a low-frequency area $(0.02-0.06 \mathrm{~Hz})$, a mid-frequency band $(0.07-0.14 \mathrm{~Hz})$, and a high-frequency band $(0.15-0.40 \mathrm{~Hz})[5]$. Some authors stated these ranges as the very low frequency band (VLF, $0.02-0.06 \mathrm{~Hz}$ ), the low frequency band (LF, $0.07-0.14 \mathrm{~Hz}$, known as $0.1 \mathrm{~Hz}$ component of $\mathrm{HRV}$ ), and the high frequency band $(\mathrm{HF}, 0.15-0.40 \mathrm{~Hz})$. The aforementioned frequency ranges also have been slightly altered by some researchers. In spectral analysis, LF variations reflect 
sympathetic and parasympathetic activities, while HF variations reflect parasympathetic activity. $\mathrm{LF} / \mathrm{HF}$ ratio, therefore, indicates overall balance between SNS and PNS. Indices of SNS ( $\left.\mathrm{I}_{\mathrm{SNS}}\right)$ and PNS $\left(\mathrm{I}_{\mathrm{PNS}}\right)$, as normalized powers of LF and HF also have been used as measures of autonomic nervous activity in response to mental and physical demands [8]. These normalized powers of LF and HF are calculated using total power (TP, $0-0.4 \mathrm{~Hz}$ ) and VLF as follows: $\mathrm{I}_{\mathrm{SNS}}=\mathrm{LF} /(\mathrm{TP}-\mathrm{VLF})$ and $\mathrm{I}_{\mathrm{PNS}}=\mathrm{HF} /(\mathrm{TP}-\mathrm{VLF})$.

Although HRV technique has shown correlations with mental effort in many studies, contradictory results have been reported [9]. Nickel and Nachreiner [10] argued against using HRV as a measure of mental and cognitive workloads. Results from their study did not support acceptable sensitivity and diagnosticity of $0.1 \mathrm{~Hz}$ component of HRV as an indicator of mental strain. Engström et al. [11] tried to differentiate the effects of visual and cognitive load on driving performance and driver state in motorway driving. They examined an in-vehicle information system with different levels of difficulty. No main effects were found on HRV in a moving base simulator.

A research program was undertaken to investigate how task automation in agricultural semi-autonomous vehicles influenced drivers' mental workload. Multiple mental workload measurement techniques were used in a simulator study. This paper describes heart rate variability in response to task automation with the intent of assessing its sensitivity to task automation.

\section{METHODOLOGY}

\subsection{Participants}

30 university students (28 males, 2 females) with at least one year (season) of agricultural tractor driving experience, mostly from farm families, participated in the experiments. Mean tractor driving experience was $7.7 \mathrm{yr}$ (minimum $1 \mathrm{yr}$, maximum $14 \mathrm{yr}$ ) meaning that all participants have been exposed to the driving task for many hours and were highly experienced. Only four of them had less than $5 \mathrm{yr}$ tractor driving experience. Participants were young, ranging in age from 18 to $25 \mathrm{yr}(\mathrm{M}=20.93)$. In case of ethnicity, there were 28 Caucasian and 2 Chinese participants. None of them had prior experience with the current version of the tractor driving simulator (TDS) located in the Agricultural Ergonomics Laboratory at the University of Manitoba. Only one subject had participated in a study with the previous version of TDS. Participants were compensated monetarily upon completion of the experiment.

\subsection{Apparatus}

The stationary tractor driving simulator (TDS) located in the Agricultural Ergonomics Laboratory at the University of Manitoba was used for conducting the experiments. Consisting of a real tractor cab, TDS provides an excellent test environment for studying drivers' behavior in agricultural vehicles. An information display inside the cab provides the necessary information to drivers, enabling them to monitor and control the implement parameters and the tractor location in the field. Three projectors project a naturalistic scene view on a curved screen in front of the cab. Two monitors behind the cab are used to simulate working units of an implement. In this study, the TDS was used to simulate a tractor air-seeder system that is used to place seeds and fertilizer in soil. Photographs of the simulator are shown in Fig. (1).

HR data were recorded using the Polar S810 heart rate monitor (Polar Electro, Finland). HRV data recordings were made over driving blocks. At the end of each trial, data were transferred to a computer. Polar precision performance software was used to analyze the data. Time and frequency domain parameters were calculated over $10 \mathrm{~min}$ of the driving blocks. Data from the first 90 s and last 30 s of driving blocks were ignored.

\subsection{Driving Task}

Tractor air-seeder system operation involves i) driving and ii) implement control and monitoring tasks. In manual setup, drivers need to perform both tasks simultaneously. The driving task requires the operator to turn the simulator's steering wheel to guide the vehicle through the field. A mapping system provides an overhead view of the portion of the field covered to help the driver avoid gaps and overlaps between adjacent passes. The air-seeder control and monitoring task is a supervisory task in which the operator needs to constantly monitor the air-seeder parameters using the information display (mounted to the right of the steering wheel) and make adjustments using the control panel to the right of the operator's seat when required. The simulator displays the following parameters: seed and fertilizer levels in containers, seed and fertilizer application rates, fan rotational speed, seeding depth, tool pressure and working speed. The operator's objective is to maintain each parameter within an acceptable performance range.

In order to evaluate the effects of automation, an automatic steering system was considered besides manual steering of the TAS. In addition, five different degrees of automation were applied to the air-seeder control and monitoring task. Automation conditions were adapted from a model for types and levels of automation presented by Parasuraman et al. [12]. This model, which is based on information processing functions, breaks down a task to four functions of (a) information acquisition (sensing), (b) information analysis (perceiving), (c) decision selection (decision-making), and (d) action implementation (actiontaking). Different levels of automation, from no automation to completely automatic, can be applied to such functions. Besides a manual mode (i.e., no automation support), the automation modes that were created for the air-seeder control and monitoring task included information acquisition support, information analysis support, decision support, and action implementation support modes.

In information acquisition mode, the automated system supported drivers by highlighting the parameter that needed adjustment. A flashing box appeared around the parameter icon immediately after it went beyond the acceptable working range. In the case of information analysis support, a message was added to the flashing box to notify the operator. Decision support mode was very similar to the information analysis support mode, with the difference being in the type of the message. In this case, the message offered a solution for the error. Finally, action implementation mode was a situation in which the computer corrected errors itself immediately after their emergence. A message notified 

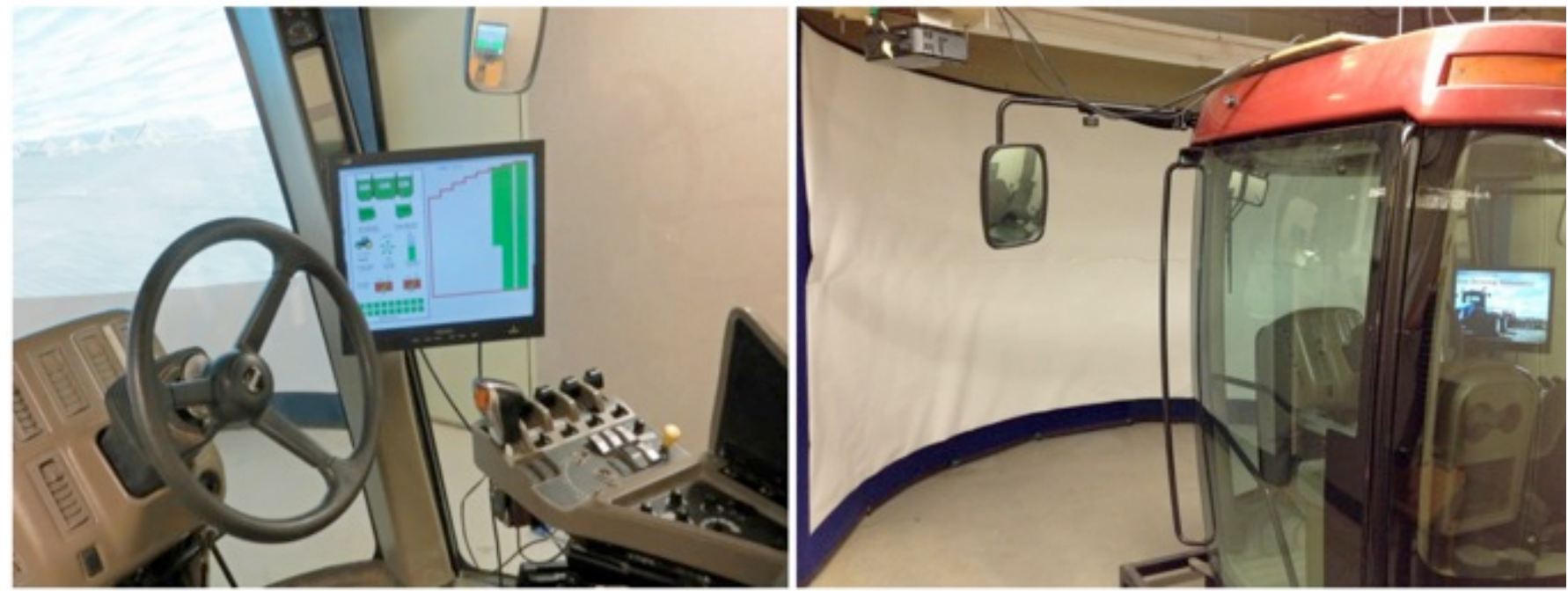

Fig. (1). The TDS and its components.

operators about the action. It was assumed that mental workload would follow a decreasing trend as automation was introduced at each of these four modes (i.e., from sensing to action-taking).

\subsection{Experimental Design and Procedure}

According to the experimental condition and based on previous studies, subjects and driving blocks were two blocking factors. A repeated Latin square design was used to avoid a learning effect and to accommodate the limited number of subjects (considering the number of treatments). The experiment included six $5 \times 5$ Latin squares, sharing same columns (driving period), with rows of subjects. Subjects were randomly assigned to the groups. For each group, only one steering mode (manual or auto-steer) was assigned, so there were three groups in manual steering mode and three groups in automatic steering mode. Automation support, as the within-subject design, was assigned to each participant. Data were analyzed using SAS statistical software (SAS Institute Inc., Cary, North Carolina, USA). The assumption of normality was verified by examining residual plots. Effects were considered highly significant at the 0.05 level.

The experiment required approximately $2 \mathrm{hrs}$ of participation. Explanations of the test procedure and necessary instructions were provided to participants upon arrival to the Lab. They were required to sign a consent letter containing such information. They then completed a $15 \mathrm{~min}$ training session where they learned to drive the simulator and to perform the control and monitoring task of the airseeder. Afterwards, they completed the five experimental driving scenarios, each $12 \mathrm{~min}$ in duration. The experimental methodology received approval by the University of Manitoba Human Ethics Board.

\section{RESULTS}

\subsection{HRV}

Various HR and HRV parameters were considered in this study. Time domain parameters involved: 1) number of heartbeats (bpm), 2) minimum RR Interval (ms), 3) average RR Interval (ms), 4) maximum RR Interval (ms), 5) standard deviation (ms), 6) $\mathrm{max} / \mathrm{min}$ ratio, 7) $\mathrm{RMSSD}(\mathrm{ms}$ ), and 8) pNN50 (\%). RMSSD is the root-mean square of differences of successive RR intervals. pNN50 is the percent of differences of adjacent RR intervals greater than $50 \mathrm{~ms}$. Parameters involved in the frequency domain were: 1) total power, TP $(0.00-0.40 \mathrm{~Hz})$, a short-term estimate of the total power of power spectral density, 2) very low frequency, VLF $(0.00-0.07 \mathrm{~Hz})$ which indicates overall activity of various slow mechanisms of sympathetic function, 3) low frequency, LF $(0.07-0.14 \mathrm{~Hz})$, that reflects both sympathetic and parasympathetic activity, 4) high frequency, $\mathrm{HF}(0.14-0.40 \mathrm{~Hz})$ that reflects parasympathetic activity, and 5) $\mathrm{LF} / \mathrm{HF}$ ratio that indicates overall balance between sympathetic and parasympathetic systems.

For the statistical analysis, most of the parameters required transformation. Depending on the data values, logarithmic or square-root transformations were used to normalize data and correct the skewness in distribution of variance of parameters. Table 1 shows ANOVA results for these parameters. Automation support effect was found on some of the time and frequency domain parameters. The taskload-automation interaction effect only was observed on two frequency domain parameters.

Minimum ( $\min$ ) RR Interval is the shortest interval between consecutive heartbeats during $10 \mathrm{~min}$ of driving blocks. This parameter was affected by the automation support. The changes in min RR interval in different automation support modes are shown in Fig. (2). Min RR interval decreased as the level of support increased, except for the action implementation $(636 \pm 12 \mathrm{~ms})$ mode that resulted in a longer min RR interval than decision making $(630 \pm 14 \mathrm{~ms})$ mode. ANOVA revealed significant differences among manual (656 $\pm 13 \mathrm{~ms})$, information acquisition (649 $\pm 13 \mathrm{~ms})$, information analysis $(645 \pm 13$ $\mathrm{ms}$ ) and decision-making modes. No significant difference was found between decision and action modes.

Max/min RR intervals ratio in different automation support levels is shown in Fig. (2). The general trend was increasing by the level of automation support. No significant difference was found between manual $(1.58 \pm 0.03)$ and information acquisition $(1.58 \pm 0.03)$ modes. Similarly, 
Table 1. ANOVA table for HR and HRV parameters.

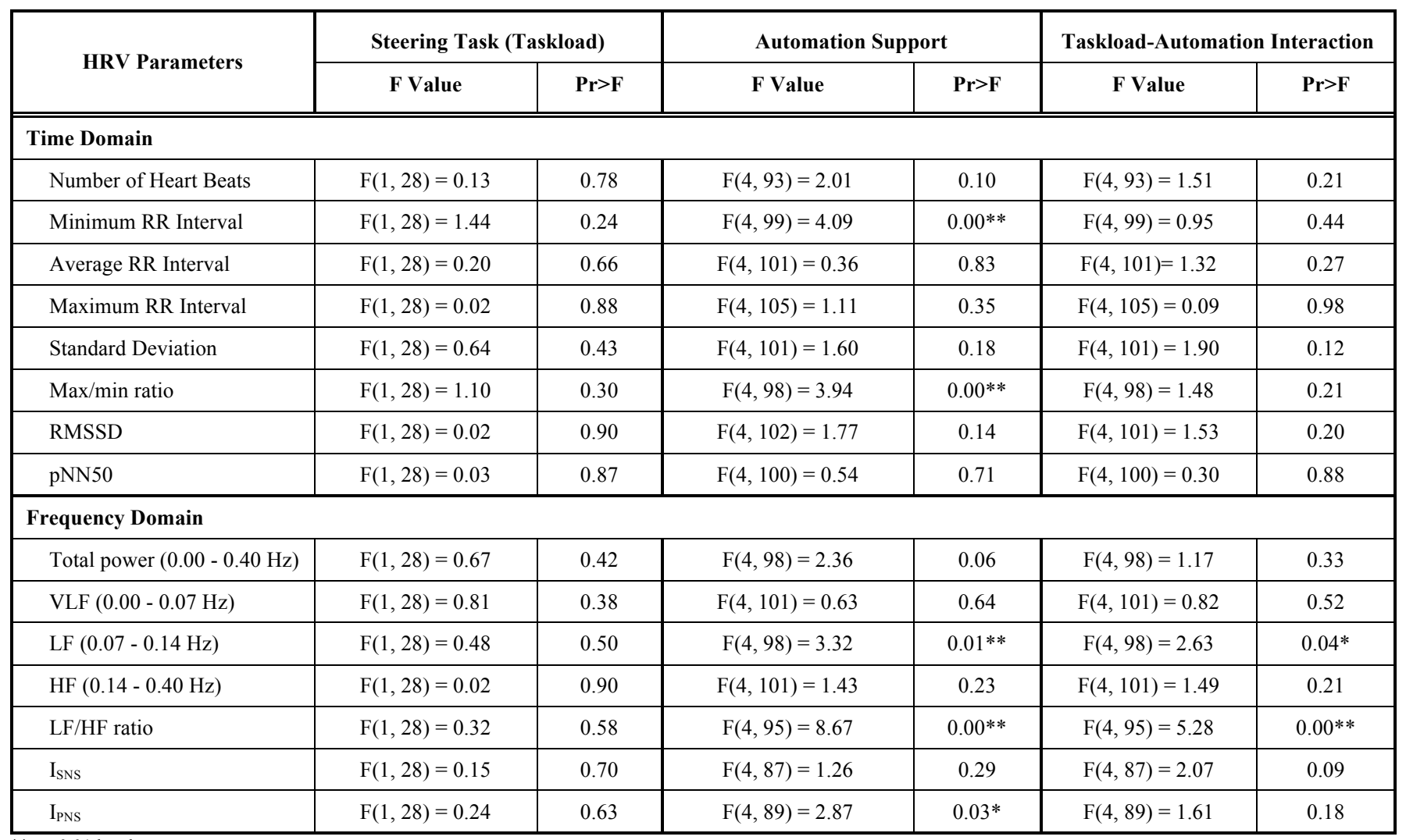

$* * p<0.01$ level.

$* p<0.05$ level.

information analysis $(1.64 \pm 0.04)$ and decision support $(1.64$ \pm 0.03 ) modes also had similar effects on the $\mathrm{max} / \mathrm{min}$ ratio. Action implementation mode $(1.67 \pm 0.04)$ resulted in the highest value.

The changes in the $0.1 \mathrm{~Hz}$ component of HRV are shown in Fig. (3). Manual steering mode resulted in lower values for LF compared to automatic steering mode; however, the differences were not statistically significant. The automation support showed a significant effect on LF. It was observed that by increasing the automation support level, the $0.1 \mathrm{~Hz}$ component was decreasing up until the decision support mode. The action implementation support mode increased the LF value. ANOVA did not reveal any differences among manual $\left(1226 \pm 149 \mathrm{~ms}^{2}\right)$, information acquisition (1147 \pm $\left.131 \mathrm{~ms}^{2}\right)$, information analysis $\left(1093 \pm 126 \mathrm{~ms}^{2}\right)$, and decision making $\left(1056 \pm 135 \mathrm{~ms}^{2}\right)$ modes. On the other hand, action implementation mode $\left(1293 \pm 172 \mathrm{~ms}^{2}\right)$ had a

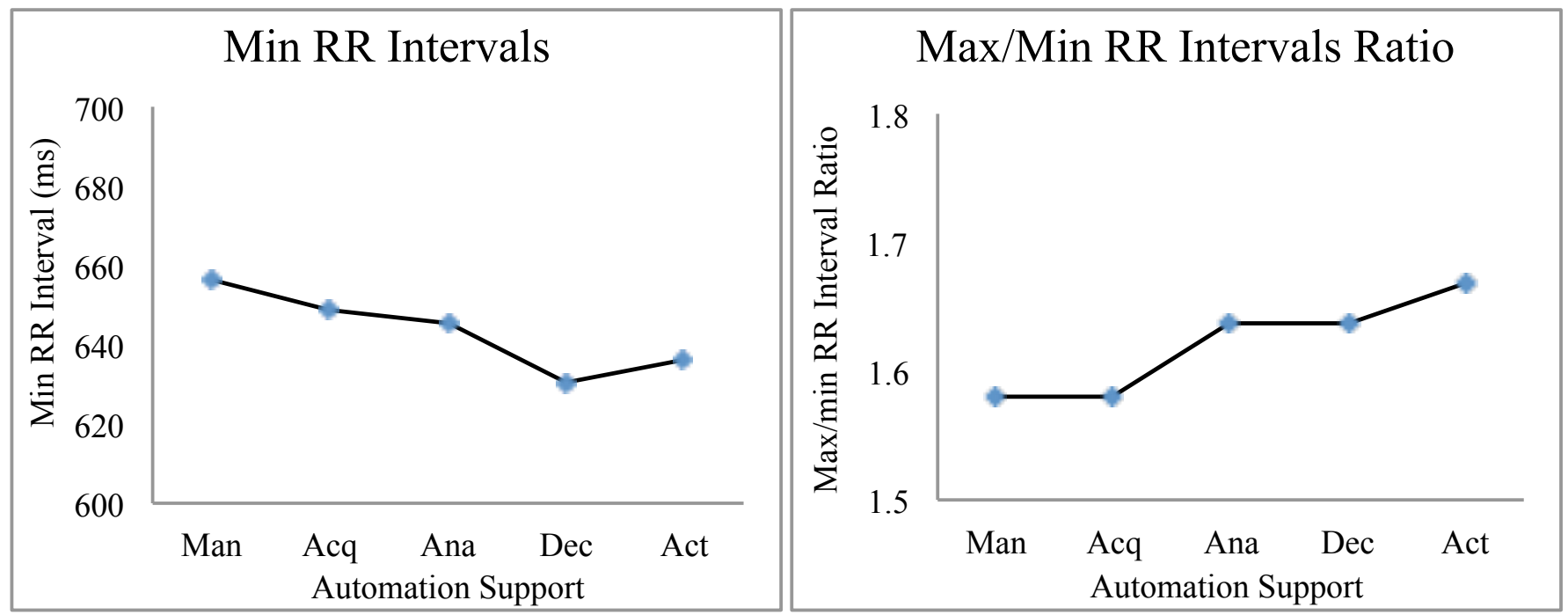

Fig. (2). Min RR intervals (left) and max/min RR intervals ratio (right) in different automation support modes: manual (Man), information acquisition (Acq), information analysis (Ana), decision making (Dec), and action implementation (Act). 
significantly different effect compared to the first four automation support modes.

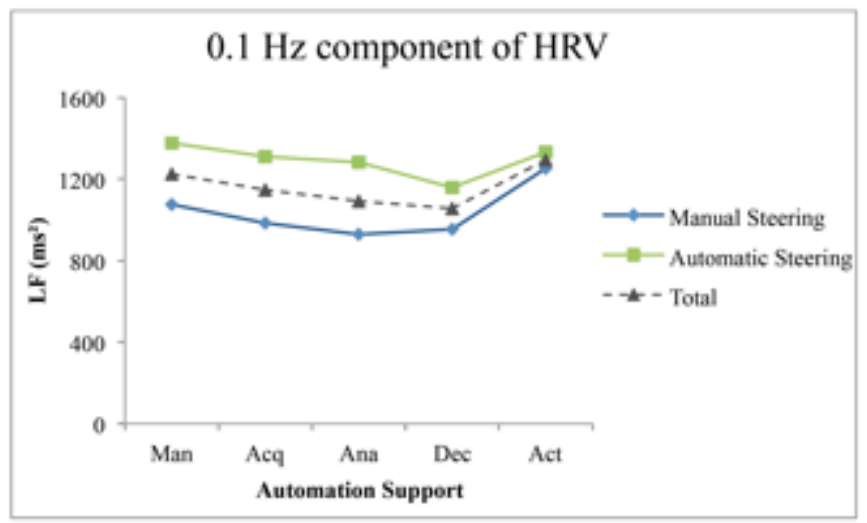

Fig. (3). The $0.1 \mathrm{~Hz}$ component of HRV in different taskload and automation conditions.

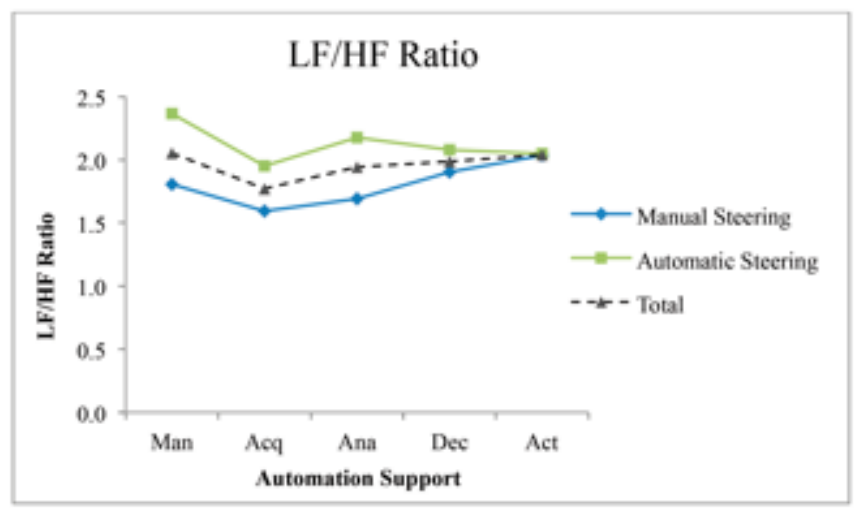

Fig. (4). The LF/HF ratio in different taskload and automation conditions.

Fig. (4) illustrates variations in $\mathrm{LF} / \mathrm{HF}$ ratio in different automation conditions. As it can be seen, LF/HF ratio remained at similar level for all of the driving blocks in automatic steering mode. In fact, dramatic changes were observed in the manual steering mode. The ANOVA for $\mathrm{LF} / \mathrm{HF}$ ratio revealed significant main effect of automation supports and taskload-automation interaction. The highest $\mathrm{LF} / \mathrm{HF}$ ratio $(0.54 \pm 0.11)$ was observed in action implementation mode while the information acquisition mode resulted in the lowest ratio $(0.41 \pm 0.12)$. Aside from the manual mode $(0.51 \pm 0.14)$, the trend was increasing as the level of automation support increased. The effect of Information acquisition mode was significantly different from all of the other automation modes. Action implementation mode also showed significant differences with all of the automation support modes except for the manual mode.

Normalized power of HF, I response to variations in level of automation support (Fig. 5). Information acquisition $(0.39 \pm 0.02)$ mode resulted in higher $\mathrm{I}_{\mathrm{PNS}}$ compared to manual mode $(0.37 \pm 0.03)$. By increasing the level of support after information acquisition mode, a decreasing trend could be observed. Despite the variations in $\mathrm{I}_{\mathrm{PNS}}$, ANOVA only showed significant differences between information acquisition mode and other automation levels. In the other words, no significant differences were found among manual, information analysis
$(0.38 \pm 0.03)$, decision support $(0.37 \pm 0.03)$ and action implementation $(0.35 \pm 0.04)$ modes.

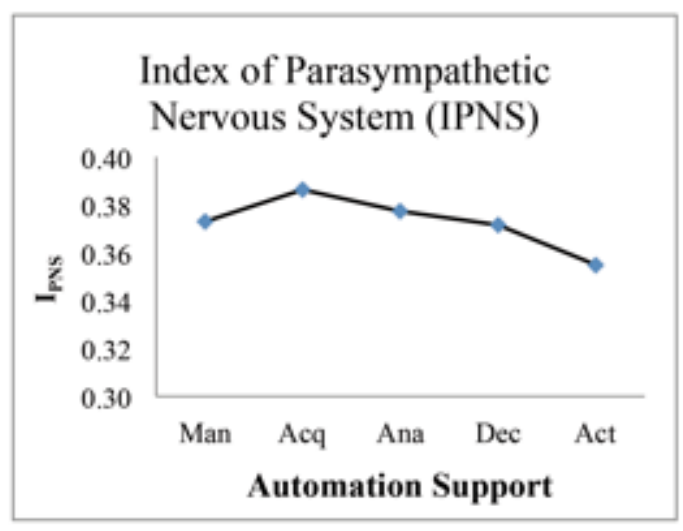

Fig. (5). Index of Parasympathetic Nervous System (IPNS) in different automation support modes.

\section{DISCUSSION}

Using a tractor driving simulator, the effect of task automation on mental workload was investigated. HRV measure was used as an indicator of workload. It was hypothesized that an increase in the level of automation of steering task and ICMT would decrease drivers' mental workload. Support for this hypothesis was found in spite of some contradictory results that could be seen in some cases. Five out of $15 \mathrm{HR}$ and HRV parameters presented changes in workload.

As the level of ICMT automation increased, MinRR interval decreased. Considering the supposedly inverse relationship between levels of automation and mental workload, the result from min RR interval is contradictory. Considering that max RR interval did not show changes to automation alterations, max/min RR intervals ratio can be a better parameter for assessing mental workload. Lower max/min RR intervals ratio has been stated to show lower parasympathetic activity [13]. Assuming the dynamically changing situation in the experiments, lower PNS activity indicates higher SNS activity and, therefore, higher mental workload.

Max/min RR intervals ratio indicated three levels of workload: higher workload in manual and information acquisition modes, medium workload in information analysis and decision selection modes, and lower workload in action implementation mode. Based on the automation conditions design, there were distinct differences among these three levels. For the first two conditions (manual and information acquisition modes), no messages were provided in the implement information display. The types of messages in the information analysis and decision-making modes were alarming, making operators aware of projected errors. In action implementation automation, only informative messages were shown to operators, indicating adjustments that were made by the machine. Therefore, providing messages to the operator and the type of message can have a great impact on mental workload.

It was observed that the highest level of automation support mode (i.e., action implementation) which put the drivers out of the ICMT loop resulted in higher LF value, 
indicating the lowest mental workload. In conditions that subjects were involved in the task-loop (i.e., manual, information acquisition, information analysis, and decision support modes), a decreasing trend (as opposed to expected increasing trend) was observed in the $0.1 \mathrm{~Hz}$ component of HRV as the level of automation increased, but the differences between the automation support modes were not statistically significant. $\mathrm{LF} / \mathrm{HF}$ ratio and $\mathrm{I}_{\mathrm{PNS}}$ showed inconsistencies. LF/HF ratio remained in a similar level for all of the automation support modes, except for a lower value in information acquisition mode. $\mathrm{I}_{\mathrm{PNS}}$ values were also at a similar level for all of the automation support conditions, except for the higher value in information acquisition mode.

The insensitivity of $0.1 \mathrm{~Hz}$ component of $\mathrm{HRV}$, which is widely used for mental workload assessment, to mental workload variations in the first four automation support modes, as well as insensitivity of the other frequency domain parameters in this study can be due to several reasons. The "globalness" of the measure has been stated as one of the reasons for finding no effect of mental load on HRV [14]. A study by Lee and Park [15] showed that an increase in physical load increased HR and decreased HRV. However, in their experiment, increase in mental load reduced HRV but had no effect on HR. Hjortskov et al. [16] stated that the characteristic of the experimental stressor may be the reason for a lack of association between HRV and mental stress. Garde et al. [8] found variations in $\mathrm{I}_{\mathrm{SNS}}$ and $\mathrm{I}_{\mathrm{PNS}}$ in response to a physically demanding reference computer task. They did not observe any effect of additional mental demands on these parameters. They concluded that physical demands significantly influenced $I_{S N S}$ and $I_{P N S}$ rather than mental demands during computer work. Other factors that affect HR include muscular fatigue and anxiety [17].

The study had several limitations that likely affected the results. First, from results and observations it was clear that there was variability among participants with respect to their skills and confidence in performing trials. Second, time of day for each individual varied by his/her availability. Participants could choose between morning or afternoon sessions. Next, the training session was short compared to the amount of time that an operator needs to become accustomed to a new system. Similarly, driving blocks were much shorter than real world operations.

\section{CONCLUSION}

The measurement of operators' mental workload in agricultural vehicles allows better understanding of the interactions between operators and the automated systems they use. Physiological measure of HRV, if sensitive enough, can be a very useful tool for this purpose. An experiment was performed to assess the effect of steering task and ICMT automation support on the tractor driver's mental workload and performance parameters using a tractor driving simulator. Although some parameters of HRV showed sensitivity to changes in driving conditions, in most cases, HRV was unable to differentiate mental workload levels. When the drivers were involved in the task-loop, the widely used HRV parameter, $0.1 \mathrm{~Hz}$ component of HRV, was not sensitive enough to differentiate mental workload levels. More studies will be needed to assess the sensitivity of the HRV measure to mental workload changes due to automation in agricultural practice, as there were certain limitations in this study. Future studies should consider all of the parameters that can affect HRV. The duration of training session and driving blocks should be long enough to present long hours of driving on a field. Moreover, different types of implements should be used in future experiments to be able to generalize the results.

\section{CONFLICT OF INTEREST}

The authors confirm that this article content has no conflicts of interest.

\section{ACKNOWLEDGEMENTS}

The authors acknowledge the financial assistance of the Natural Sciences and Engineering Research Council of Canada (NSERC). We also thank G. H. Crow, L. Onischuk, H. Khazanehei, and M. Porter for their support and CaseNewHolland for donating a modern tractor cab to be used in the simulator.

\section{REFERENCES}

[1] Di Stasi LL, Antolí A, Cañas JJ. Evaluating mental workload while interacting with computer-generated artificial environments. Entertain Comput 2013; 4(1): 63-9.

[2] Gao Q, Wang Y, Song F, Li Z, Dong X. Mental workload measurement for emergency operating procedures in digital nuclear power plants. Ergonomics 2013; 56(7): 1070-85.

[3] Brookhuis KA, de Waard D. Monitoring drivers' mental workload in driving simulators using physiological measures. Accid Anal Prev 2010; 42(3): 898-903.

[4] Lei S, Roetting M. Influence of task combination on EEG Spectrum Modulation for driver workload estimation. Hum Factors 2011; 53(2): 168-79.

[5] Mulder LJM, de Waard D, Brookhuis KA. Estimating mental effort using heart rate and heart rate variability. Handbook of Human Factors and Ergonomics Methods. USA: CRC Press 2004.

[6] Rajendra AU, Paul JK, Kannathal N, Lim CM, Suri JS. Heart rate variability: a review. Med Biol Eng Comput 2006; 44(12): 103151.

[7] Sato N, Miyake S. Cardiovascular reactivity to mental stress: relationship with menstrual cycle and gender. J Physiol Anthropol Appl Hum Sci 2004; 23(6): 215-23.

[8] Garde A, Laursen B, Jørgensen A, Jensen B. Effects of mental and physical demands on heart rate variability during computer work. Eur J Appl Physiol 2002; 87(4-5): 456-61.

[9] Li P, Wu B, Zhang Y, et al. Changes in heart rate variability during manual controlled rendezvous and docking with task complexity. In: Duffy V, Ed. Digital Human Modeling and Applications in Health, Safety, Ergonomics, and Risk Management Human Body Modeling and Ergonomics. Lecture Notes in Computer Science. 8026. Berlin, Heidelberg: Springer 2013; pp. 86-92.

[10] Nickel P, Nachreiner F. Sensitivity and diagnosticity of the $0.1-\mathrm{Hz}$ component of heart rate variability as an indicator of mental workload. Hum Factors. 2003; 45(4): 575-90.

[11] Engström J, Johansson E, Östlund J. Effects of visual and cognitive load in real and simulated motorway driving. Transp Res Part F Traffic Psychol Behav 2005; 8(2): 97-120.

[12] Parasuraman R, Sheridan TB, Wickens CD. A model for types and levels of human interaction with automation. IEEE Trans Syst Man Cybern Syst 2000; 30(3): 286-97.

[13] Nayem M, Begum N, Ferdousi S. Time domain measures of heart rate variability to assess autonomic dysfunction in irritable bowel syndrome. J Bangladesh Soc Physiol 2013; 7(2): 60-5.

[14] De Waard D. The measurement of drivers' mental workload. PhD Dissertation. Haren, Netherlands: University of Groningen 1996. 
[15] Lee DH, Park KS. Multivariate analysis of mental and physical load components in sinus arrhythmia scores. Ergonomics 1990; 33(1): 35-47.

[16] Hjortskov N, Rissén D, Blangsted A, et al. The effect of mental stress on heart rate variability and blood pressure during computer work. Eur J Appl Physiol 2004; 92(1-2): 84-9.
[17] Borghini G, Astolfi L, Vecchiato G, Mattia D, Babiloni F. Measuring neurophysiological signals in aircraft pilots and car drivers for the assessment of mental workload, fatigue and drowsiness. Neurosci Biobehav R 2014; 44: 58-76.

(C) Bashiri and Mann; Licensee Bentham Open.

This is an open access article licensed under the terms of the Creative Commons Attribution Non-Commercial License (http://creativecommons.org/licenses/bync/3.0/) which permits unrestricted, non-commercial use, distribution and reproduction in any medium, provided the work is properly cited. 